\title{
Erratum: Using surface impedance for calculating wakefields in flat geometry [Phys. Rev. ST Accel. Beams 18, 034401 (2015)]
}

\author{
K. Bane and G. Stupakov \\ (Received 27 January 2016; published 24 March 2016)
}

DOI: $10.1103 /$ PhysRevAccelBeams.19.039901

Our derivation of the impedance in Sec. II of the paper used a relation $i k E_{z}=\partial H_{x} / \partial y$ between the longitudinal electric field and the component of the magnetic field $H_{x}$. This relation is incorrect; the correct one is $i k E_{z}=\partial H_{x} / \partial y-\partial H_{y} / \partial x$, as follows from the Maxwell equation $i k \boldsymbol{E}=\nabla \times \boldsymbol{H}$ in Fourier representation. This flaw in the derivation, however, was compensated when we subtracted, in Eq. (12), the contribution for the longitudinal impedance for perfectly conducting walls. Our final result of this section, Eqs. (13) and (14), as well all the subsequent results in the paper are correct.

In Ref. [1] we worked out a different, rigorous derivation of Eqs. (13) and (14) starting from Maxwell's equation. The new derivation reproduces Eqs. (13) and (14) and also provides conditions, not mentioned in our paper, when these equations are valid. They are: $|\zeta| \ll 1$, where $\zeta$ is the surface impedance, and $k \gg 1 / a$ where $k$ is the wave number and $a$ is the half-gap of the structure. While the first of these conditions is easily satisfied in most applications, the second one means that our results are only applicable for short bunches, when the bunch length $\sigma_{z} \ll a$. Note that the focus of our paper was on short bunches typical for modern free electron lasers; the condition $\sigma_{z} \ll a$ in these applications is usually satisfied as well.

[1] K. Bane and G. Stupakov, SLAC Report No. SLAC-PUB-16457, 2016.

Published by the American Physical Society under the terms of the Creative Commons Attribution 3.0 License. Further distribution of this work must maintain attribution to the author(s) and the published articles title, journal citation, and DOI. 